\title{
Viscosity B-Coefficient for Sodium Chloride in Aqueous Mixtures of 1,4-Dioxane at Different Temperatures
}

\author{
Renato Tomaš, ${ }^{1, *}$ Toni Jovanović ${ }^{1}$ and Marija Bešter-Rogač² \\ ${ }^{1}$ Faculty of Chemistry and Technology, University of Split, Nikole Tesle 10, HR-21000 Split, Croatia. \\ ${ }^{2}$ Faculty of Chemistry and Chemical Technology, University of Ljubljana, Večna pot 113, SI-1000 Ljubljana, Slovenia. \\ *Corresponding author: E-mail: rtomas@ @tf-split.hr
}

Received: 19-02-2015

Dedicated to prof. Jože Koller on the occasion of his $70^{\text {th }}$ birthday.

\begin{abstract}
Viscosities of sodium chloride solutions in water-1,4-dioxane binary mixtures with mole fractions of 1,4-dioxane, $x_{\mathrm{D}}=$ $0.05,0.10,0.15$ and 0.20 , in the temperature range from 278.15 to $318.15 \mathrm{~K}$ were determined. The relative viscosity data have been analyzed and interpreted in terms of the rearranged Jones-Dole equation, $\left(\eta_{\mathrm{r}}-1\right)-A c^{1 / 2}=B c$. The viscosity $A$-coefficients were calculated from Falkenhagen and Vernon theory by help of the literature limiting ionic conductivity data and $B$-coefficients were estimated from linear plots. All $B$-coefficients obtained for $\mathrm{NaCl}$ water-1,4-dioxane binary mixtures are positive at all temperatures. These data were compared with the data for $\mathrm{NaCl}$ in aqueous medium at different temperatures reported in literature. The ion-ion, ion-solvent and solvent-solvent interactions have been discussed.
\end{abstract}

Keywords: Sodium chloride, water-1,4-dioxane binary mixtures, viscosity data, viscosity $B$-coefficient, ions-solvent interactions

\section{Introduction}

Viscometric studies of electrolyte solutions have been often used in order to obtain information regarding solute-solvent interactions. Whereas viscosity of electrolyte solutions has been widely studied in water and organic solvents, ${ }^{1,2}$ less consideration has been devoted to solutions in solvent mixtures. A survey of the literature on the viscosity data of electrolytes solutions in aqueous mixtures with organic solvents shows that they were determined mostly for 1,1 type of electrolytes and usually at one or only several temperatures in aqueous mixtures of formamide ${ }^{3,4} \mathrm{~N}, \mathrm{~N}$-dimethylformamide, ${ }^{5}$ dimethylsulfoxide, ${ }^{6}$ acetone, ${ }^{7}$ dimethylacetamide, ${ }^{8}$ 2-ethoxyethanol, ${ }^{9}$ methanol, ${ }^{10,11}$ ethylene glycol ${ }^{12,13}$ and tert-butanol. ${ }^{14}$

Among aqueous mixtures water-1,4-dioxane mixtures permit the widest variation of the solvent permittivity ranging from 2.21 to 78.36 in pure 1,4-dioxane and in water at $298.15 \mathrm{~K}$, respectively. ${ }^{15}$ Thus, these mixtures have been used as a favourite solvent system to study ion-ion, solvent-ion and solvent-solvent interaction. ${ }^{16-19}$ Surpri- singly, there is a lack of the viscometric studies of electrolyte solutions in these mixtures. To our best knowledge there are only two investigation dealing with viscosity of electrolytes in water-1,4-dioxane mixtures. In 1973 Fuoss and Reynolds reported viscosity data for alkali metal halides and tetraalkylammonium salts in water-1,4-dioxane mixtures containing 0-63 mass \% of 1,4-dioxane at 298.15 $\mathrm{K}^{20}$ Das and Das investigated viscosities of $\mathrm{NaCI}, \mathrm{NaBr}$, $\mathrm{NaNO}_{3}, \mathrm{KCI}, \mathrm{KBr}$ and $\mathrm{KNO}_{3}$ solutions in water-1,4-dioxane mixtures with 10,20 and 30 mass $\%$ of 1.4-dioxane, and at four ranging from 303.15 to $328.15 \mathrm{~K}^{21}$ Unfortunately in this paper no experimental data are given.

In the present work precise viscometric data for $\mathrm{Na}$ $\mathrm{Cl}$ in water-1,4-dioxane binary mixtures (with mole fraction of 1,4-dioxane, $x_{\mathrm{D}}=0.05,0.10,0.15$, and 0.20 ) in the concentration range $0.05 \leq \mathrm{c} /$ moldm $^{-3} \leq 0.30$ at nine temperatures (from 278.15 to $318.15 \mathrm{~K}$ in step of $5 \mathrm{~K}$ ) are reported. The relative viscosity data, $\eta_{\mathrm{r}}$, have been analysed and interpreted in terms of the rearranged Jones-Dole equation, $\left(\eta_{\mathrm{r}}-1\right)-A c^{1 / 2}=B c$. From the reported limiting ionic conductivity data, ${ }^{16,17}$ the viscosity $A$-coefficients 
have been calculated from Falkenhagen and Vernon theory. ${ }^{2,22}$ Viscosity $B$-coefficient were determined, compared with those reported for $\mathrm{NaCl}$ in water ${ }^{2}$ and in water-1,4dioxane mixtures at $298.15 \mathrm{~K}^{20}$ and discussed in terms of ion-solvent interactions.

\section{Experimental}

\section{1. Materials}

1,4-dioxane (Merck, p.a.) was used without further purification. Demineralized water was bi-distilled in a quartz apparatus (DESTAMAT Bi18E, Heraeus). The final product with a specific conductivity of less than $0.5 \mu \mathrm{S}$ $\mathrm{cm}^{-1}$ was applied. Sodium chloride $(\mathrm{NaCl}$, Merck, p.a., $\geq 99.5 \%$ ) was dried before use at $130{ }^{\circ} \mathrm{C}$ for $24 \mathrm{~h}$. The mixed solvents were prepared by weighing and stock solutions were prepared by adding of weighed amount of solvent to weighed amount of $\mathrm{NaCl}$. Solutions of definite molality were prepared by weighing the pure mixed solvent and the stock solution of $\mathrm{NaCl}$ in a particular mixed solvent. The molarity, $c$ can be determined from the molality and density data by means of the relation:

$$
c=\frac{1000 \rho m}{(1000+m M)}
$$

where $m$ is the molality of solution (moles of electrolyte per kilogram of solvent), $\rho$ is the density of solution, and $M$ is the molar mass of sodium chloride. Taking into account the sources of errors (impurities, weighing, ...), concentrations $c$ are accurate $\pm 0.0001 \mathrm{~mol} \mathrm{dm}^{-3}$.

\section{2. Viscosity and Density Measurements}

The viscosites of solutions were determined with a micro Ubbelohde viscometer (SI Analytics $\mathrm{GmbH}$, Mainz, Germany, type and capillary no. 53610/I) and an automatic flow time measuring system ViscoSystem ${ }^{\circledR}$ AVS 370. The viscometer was immersed in a transparent thermostat bath with the precision of the set temperature better than $0.01 \mathrm{~K}$. Each measurement was automatically repeated at least five times and yielded a reproducibility of the flow time of better than $0.02 \%$. The kinematic viscosity of solutions, $v\left(\mathrm{~m}^{2} \mathrm{~s}^{-1}\right)$, was calculated from the equation $^{23} v=C \cdot t-E / t^{2}$, where $t$ (s) is the flow time, $C$ and $E$ are constants characteristic for the viscometer and were determined by calibration of the viscometer with water. The values of $C=1.00772 \times 10^{-8} \mathrm{~m}^{2} \mathrm{~s}^{-2}$ and $E=$ $-4.9602 \times 10^{-5} \mathrm{~m}^{2} \mathrm{~s}$ were determined by using the kinematic viscosity of water $\left(v(293.15 \mathrm{~K})=1.0038 \times 10^{-6} \mathrm{~m}^{2}\right.$ $\mathrm{s}^{-1}$ and $\left.\left.v(298.15 \mathrm{~K})=0.8929 \times 10^{-6} \mathrm{~m}^{2} \mathrm{~s}^{-1}\right)\right)^{24}$ and the flow times at $293.15 \mathrm{~K}$ and $298.15 \mathrm{~K}$. The absolute (dynamic) viscosity, $\eta\left(\mathrm{Pa} \mathrm{s}=\mathrm{kg} \mathrm{m}^{-1} \mathrm{~s}^{-1}\right)$, was obtained from the relation, $\eta=v \rho$, where $\rho\left(\mathrm{kg} \mathrm{m}^{-3}\right)$ is the density of investigated solution, which was determined at each tempe- rature $( \pm 0.005 \mathrm{~K})$ by the method of Kratky et al. ${ }^{25}$ using Anton Paar density meter DMA 5000 (Anton Paar, Graz, Austria) with a declared reproducibility $\sim 5 \times 10^{-3} \mathrm{~kg} \mathrm{~m}^{-3}$. The errors from calibration and temperature control yield an uncertainty of $0.05 \%$ of absolute viscosity.

\section{Results and Discussion}

The measured viscosity, $\eta$, and density, $\rho$, data for the solutions of $\mathrm{NaCl}$ at all operating temperatures in water-1,4-dioxane binary mixtures with mole fraction of dioxane, $x_{\mathrm{D}}=0.05,0.10,0.15$, and 0.20 are presented in Tables 1-4, and in Figures S1 - S4 in Supporting information. It is evident that density and viscosity continually increase with increasing concentration of $\mathrm{NaCl}$ and increasing content of 1,4-dioxane in the mixture and decreases with increasing temperature in all investigated systems.

A comparison between densities, $\rho_{0}$, and viscosities, $\eta_{0}$, of the solvent mixtures as determined in our work and the literature data ${ }^{16,26,27}$ at 293.15 and $303.15 \mathrm{~K}$ shows an excellent agreement (Figure S5 in Supporting information).

The relative viscosity, $\eta_{\mathrm{r}}$, of electrolyte solution is related to the molarity, $c$, according to the Jones-Dole equation:

$$
\eta_{\mathrm{r}}=\eta / \eta_{0}=1+A c^{1 / 2}+B c
$$

where $\eta$ and $\eta_{0}$ are the viscosities of the solution and solvent, respectively. Eq. (1) in this form, is used by the most of authors for processing viscosity data. ${ }^{22,28}$ At higher concentration $\left(c>0.5 \mathrm{~mol} \mathrm{dm}^{-3}\right)$ a quadratic term $D c^{2}$ is added in eq. (2) and that expression is known as $\mathrm{Ka}$ minsky relation. ${ }^{29}$ Viscosity coefficients $A, B$, and $D$ depend on the solute, the solvent, and the temperature.

The viscosity $A$-coefficient is a measure of long-range ion-ion interactions, and the viscosity $B$-coefficient depends on ion-solvent interactions. ${ }^{2,30}$ The $A$ and $B$ coefficients can be obtained from the intercept and the slope of the straight line using Eq. (2) in the next form

$$
\left(\eta_{\mathrm{r}}-1\right)-A \cdot c^{1 / 2}=B \cdot c
$$

Thus determined $A$-values should be positive, while $B$-values can be positive or negative. Furthermore, if the limiting molar conductivities of the constituent ions are known, $A$-value can be calculated theoretically from the ionic interaction theory by Falkenhagen and Vernon ${ }^{2,22}$ by help of the relation ${ }^{28}$

$$
A=\frac{0.2577 \Lambda^{\infty}}{\eta_{0}\left(\varepsilon_{\mathrm{r}} T\right)^{1 / 2} \lambda_{+}^{\infty} \lambda_{-}^{\infty}}\left[1-0.6863\left(\frac{\lambda_{+}^{\infty}-\lambda_{-}^{\infty}}{\Lambda^{\infty}}\right)^{2}\right]
$$

where $\varepsilon_{\mathrm{r}}$ is relative permittivity of the solvent and $\Lambda^{\infty}, \lambda_{+}^{\infty}$, and $\lambda_{-}^{\infty}$, are limiting molar conductivities of the electrolyte, cation, and anion respectively, at temperature $T$. 
Table 1. Experimental densities, $\rho$, and viscosities, $\eta$, as function of concentration, $c$, of $\mathrm{NaCl}$ solution in water-1,4-dioxane mixtures with mole fraction of 1,4- dioxane, $x_{\mathrm{D}}=0.05$ at different temperatures.

\begin{tabular}{|c|c|c|c|c|c|c|c|c|}
\hline$c / \mathrm{mol} \mathrm{dm}^{-3}$ & $\rho / \mathrm{g} \mathrm{cm}^{-3}$ & $\eta / \mathrm{mPa} \mathrm{s}$ & $c / \mathrm{mol} \mathrm{dm}$ & $\rho / \mathrm{g} \mathrm{cm}^{-3}$ & $\eta / \mathrm{mPa}$ & $c / \mathrm{mol} \mathrm{dm}$ & $\rho / \mathrm{g} \mathrm{cm}^{-3}$ & $\eta / \mathrm{mPa} s$ \\
\hline \multicolumn{3}{|c|}{$T=278.15 \mathrm{~K}$} & \multicolumn{3}{|c|}{$T=293.15 \mathrm{~K}$} & \multicolumn{3}{|c|}{$T=308.15 \mathrm{~K}$} \\
\hline 0 & 1.022388 & 2.3061 & 0 & 1.016686 & 1.4614 & 0 & 1.009405 & 1.0144 \\
\hline 0.0511 & 1.024616 & 2.3238 & 0.0508 & 1.018802 & 1.4783 & 0.0504 & 1.011565 & 1.0234 \\
\hline 0.1021 & 1.026697 & 2.3438 & 0.1015 & 1.020837 & 1.4895 & 0.1008 & 1.013606 & 1.0325 \\
\hline 0.1530 & 1.028743 & 2.3577 & 0.1521 & 1.022884 & 1.4990 & 0.1510 & 1.015502 & 1.0407 \\
\hline 0.2038 & 1.030942 & 2.3689 & 0.2026 & 1.024967 & 1.5092 & 0.2012 & 1.017606 & 1.0468 \\
\hline 0.2545 & 1.032731 & 2.3798 & 0.2531 & 1.027012 & 1.5174 & 0.2512 & 1.019622 & 1.0531 \\
\hline 0.3052 & 1.035021 & 2.4047 & 0.3034 & 1.028953 & 1.5313 & 0.3012 & 1.021537 & 1.0627 \\
\hline \multicolumn{3}{|c|}{$T=283.15 \mathrm{~K}$} & \multicolumn{3}{|c|}{$T=298.15 \mathrm{~K}$} & \multicolumn{3}{|c|}{$T=313.15 \mathrm{~K}$} \\
\hline 0 & 1.020564 & 1.9555 & 0 & 1.014426 & 1.2848 & 0 & 1.006727 & 0.9131 \\
\hline 0.0510 & 1.022863 & 1.9799 & 0.0507 & 1.016517 & 1.2971 & 0.0503 & 1.008864 & 0.9207 \\
\hline 0.1019 & 1.024991 & 1.9921 & 0.1013 & 1.018598 & 1.3026 & 0.1005 & 1.010911 & 0.9297 \\
\hline 0.1527 & 1.027038 & 2.0046 & 0.1518 & 1.020603 & 1.3153 & 0.1506 & 1.012804 & 0.9355 \\
\hline 0.2034 & 1.029094 & 2.0156 & 0.2022 & 1.022659 & 1.3216 & 0.2006 & 1.014897 & 0.9400 \\
\hline 0.2540 & 1.031001 & 2.0252 & 0.2525 & 1.024688 & 1.3335 & 0.2506 & 1.016902 & 0.9484 \\
\hline 0.3046 & 1.033217 & 2.0433 & 0.3027 & 1.026653 & 1.3369 & 0.3004 & 1.018806 & 0.9554 \\
\hline \multicolumn{3}{|c|}{$T=288.15 \mathrm{~K}$} & \multicolumn{3}{|c|}{$T=303.15 \mathrm{~K}$} & \multicolumn{3}{|c|}{$T=318.15 \mathrm{~K}$} \\
\hline 0 & 1.018775 & 1.6845 & 0 & 1.011946 & 1.1262 & 0 & 1.003919 & 0.8249 \\
\hline 0.0509 & 1.020893 & 1.7031 & 0.0506 & 1.014158 & 1.1398 & 0.0502 & 1.005910 & 0.8297 \\
\hline 0.1017 & 1.022998 & 1.7134 & 0.1010 & 1.016174 & 1.1501 & 0.1002 & 1.008089 & 0.8382 \\
\hline 0.1524 & 1.025043 & 1.7242 & 0.1514 & 1.018116 & 1.1599 & 0.1502 & 1.009981 & 0.8440 \\
\hline 0.2031 & 1.027130 & 1.7339 & 0.2017 & 1.020186 & 1.1640 & 0.2001 & 1.012063 & 0.8512 \\
\hline 0.2536 & 1.029192 & 1.7449 & 0.2519 & 1.022259 & 1.1727 & 0.2499 & 1.014060 & 0.8583 \\
\hline 0.3040 & 1.031190 & 1.7596 & 0.3020 & 1.024156 & 1.1847 & 0.2995 & 1.015851 & 0.8627 \\
\hline
\end{tabular}

Table 2. Experimental densities, $\rho$, and viscosities, $\eta$, as function of concentration, $c$, of $\mathrm{NaCl}$ solution in water-1,4-dioxane mixtures with mole fraction of 1,4- dioxane, $x_{\mathrm{D}}=0.10$ at different temperatures.

\begin{tabular}{|c|c|c|c|c|c|c|c|c|}
\hline $\bar{c} / \mathrm{mol} \mathrm{dm}^{-3}$ & $\rho / \mathrm{g} \mathrm{cm}^{-3}$ & $\eta / \mathrm{mPa} \mathrm{s}$ & $c / \mathrm{mol} \mathrm{dm}$ & $\rho / \mathrm{g} \mathrm{cm}^{-3}$ & $\eta / \mathrm{mPa} s$ & $c / \mathrm{mol} \mathrm{dm}$ & $\rho / \mathrm{g} \mathrm{cm}^{-3}$ & $\eta / \mathrm{mPa} \mathrm{s}$ \\
\hline \multicolumn{3}{|c|}{$T=278.15 \mathrm{~K}$} & \multicolumn{3}{|c|}{$T=293.15 \mathrm{~K}$} & \multicolumn{3}{|c|}{$T=308.15 \mathrm{~K}$} \\
\hline 0 & 1.036924 & 2.9318 & 0 & 1.028437 & 1.8370 & 0 & 1.019026 & 1.2476 \\
\hline 0.0511 & 1.039070 & 2.9642 & 0.0507 & 1.030495 & 1.8539 & 0.0502 & 1.021071 & 1.2641 \\
\hline 0.1023 & 1.041146 & 2.9823 & 0.1014 & 1.032545 & 1.8703 & 0.1005 & 1.023104 & 1.2771 \\
\hline 0.1538 & 1.043129 & 3.0018 & 0.1525 & 1.034507 & 1.8814 & 0.1511 & 1.025053 & 1.2828 \\
\hline 0.2054 & 1.045281 & 3.0288 & 0.2037 & 1.036616 & 1.8963 & 0.2019 & 1.027136 & 1.2991 \\
\hline 0.2573 & 1.047019 & 3.0554 & 0.2552 & 1.038364 & 1.9189 & 0.2528 & 1.028901 & 1.3112 \\
\hline 0.3094 & 1.049527 & 3.0873 & 0.3069 & 1.040800 & 1.9395 & 0.3041 & 1.031284 & 1.3189 \\
\hline \multicolumn{3}{|c|}{$T=283.15 \mathrm{~K}$} & \multicolumn{3}{|c|}{$T=298.15 \mathrm{~K}$} & \multicolumn{3}{|c|}{$T=313.15 \mathrm{~K}$} \\
\hline 0 & 1.034227 & 2.4840 & 0 & 1.025398 & 1.6018 & 0 & 1.015692 & 1.1152 \\
\hline 0.0509 & 1.036303 & 2.5095 & 0.0505 & 1.027450 & 1.6202 & 0.0500 & 1.017737 & 1.1320 \\
\hline 0.1020 & 1.038374 & 2.5252 & 0.1011 & 1.029495 & 1.6342 & 0.1002 & 1.019766 & 1.1430 \\
\hline 0.1534 & 1.040348 & 2.5470 & 0.1521 & 1.031450 & 1.6430 & 0.1506 & 1.021713 & 1.1513 \\
\hline 0.2049 & 1.042483 & 2.5670 & 0.2031 & 1.033548 & 1.6604 & 0.2012 & 1.023790 & 1.1622 \\
\hline 0.2566 & 1.044226 & 2.5871 & 0.2544 & 1.035301 & 1.6837 & 0.2520 & 1.025561 & 1.1697 \\
\hline 0.3086 & 1.046706 & 2.6164 & 0.3060 & 1.037716 & 1.7030 & 0.3031 & 1.027932 & 1.1847 \\
\hline \multicolumn{3}{|c|}{$T=288.15 \mathrm{~K}$} & \multicolumn{3}{|c|}{$T=303.15 \mathrm{~K}$} & \multicolumn{3}{|c|}{$T=318.15 \mathrm{~K}$} \\
\hline 0 & 1.031380 & 2.1268 & 0 & 1.022261 & 1.4067 & 0 & 1.012259 & 1.0037 \\
\hline 0.0508 & 1.033445 & 2.1453 & 0.0504 & 1.024309 & 1.4209 & 0.0499 & 1.014306 & 1.0142 \\
\hline 0.1017 & 1.035506 & 2.1620 & 0.1008 & 1.026347 & 1.4305 & 0.0998 & 1.016334 & 1.0309 \\
\hline 0.1529 & 1.037474 & 2.1738 & 0.1516 & 1.028299 & 1.4404 & 0.1501 & 1.018279 & 1.0376 \\
\hline 0.2043 & 1.039595 & 2.1995 & 0.2025 & 1.030389 & 1.4595 & 0.2005 & 1.020354 & 1.0481 \\
\hline 0.2559 & 1.041388 & 2.2155 & 0.2536 & 1.032146 & 1.4756 & 0.2512 & 1.022129 & 1.0539 \\
\hline 0.3077 & 1.043795 & 2.2406 & 0.3050 & 1.034545 & 1.4932 & 0.3021 & 1.024490 & 1.0676 \\
\hline
\end{tabular}


Table 3. Experimental densities, $\rho$, and viscosities, $\eta$, as function of concentration, $c$ of $\mathrm{NaCl}$ solution in water-1,4-dioxane mixtures with mole fraction of 1,4- dioxane, $x_{\mathrm{D}}=0.15$ at different temperature.

\begin{tabular}{|c|c|c|c|c|c|c|c|c|}
\hline$\overline{c / \mathrm{mol} \mathrm{dm}}$ & $\rho / \mathrm{g} \mathrm{cm}^{-3}$ & $\eta / \mathrm{mPa} s$ & $c / \mathrm{mol} \mathrm{dm}^{-3}$ & $\rho / \mathrm{g} \mathrm{cm}^{-3}$ & $\eta / \mathrm{mPa} s$ & $c / \mathrm{mol} \mathrm{dm}^{-3}$ & $\rho / \mathrm{g} \mathrm{cm}^{-3}$ & $\eta / \mathrm{mPa} s$ \\
\hline \multicolumn{3}{|c|}{$T=278.15 \mathrm{~K}$} & \multicolumn{3}{|c|}{$T=293.15 \mathrm{~K}$} & \multicolumn{3}{|c|}{$T=308.15 \mathrm{~K}$} \\
\hline 0 & 1.045452 & 3.3327 & 0 & 1.035198 & 2.0895 & 0 & 1.024294 & 1.4125 \\
\hline 0.0515 & 1.047771 & 3.3636 & 0.0510 & 1.037504 & 2.1082 & 0.0505 & 1.026603 & 1.4288 \\
\hline 0.1031 & 1.049789 & 3.4111 & 0.1021 & 1.039571 & 2.1364 & 0.1011 & 1.028707 & 1.4524 \\
\hline 0.1551 & 1.051892 & 3.4473 & 0.1536 & 1.041719 & 2.1605 & 0.1520 & 1.030855 & 1.4803 \\
\hline 0.2072 & 1.054086 & 3.4884 & 0.2051 & 1.043886 & 2.1890 & 0.2030 & 1.033071 & 1.4990 \\
\hline 0.2594 & 1.056172 & 3.5226 & 0.2569 & 1.045993 & 2.2078 & 0.2543 & 1.035207 & 1.5090 \\
\hline 0.3120 & 1.058264 & 3.5661 & 0.3090 & 1.048112 & 2.2496 & 0.3059 & 1.037359 & 1.5338 \\
\hline \multicolumn{3}{|c|}{$T=283.15 \mathrm{~K}$} & \multicolumn{3}{|c|}{$T=298.15 \mathrm{~K}$} & \multicolumn{3}{|c|}{$T=313.15 \mathrm{~K}$} \\
\hline 0 & 1.042102 & 2.8262 & 0 & 1.031640 & 1.8333 & 0 & 1.020403 & 1.2638 \\
\hline 0.0513 & 1.044417 & 2.8567 & 0.0508 & 1.033946 & 1.8483 & 0.0503 & 1.022270 & 1.2864 \\
\hline 0.1028 & 1.046451 & 2.8990 & 0.1018 & 1.036027 & 1.8698 & 0.1007 & 1.024834 & 1.3051 \\
\hline 0.1546 & 1.048599 & 2.9378 & 0.1531 & 1.038175 & 1.8989 & 0.1514 & 1.026982 & 1.3228 \\
\hline 0.2065 & 1.050751 & 2.9765 & 0.2045 & 1.040356 & 1.9235 & 0.2023 & 1.029218 & 1.3328 \\
\hline 0.2586 & 1.052845 & 3.0101 & 0.2560 & 1.042247 & 1.9442 & 0.2533 & 1.031359 & 1.3544 \\
\hline 0.3110 & 1.054945 & 3.0480 & 0.3080 & 1.044601 & 1.9742 & 0.3047 & 1.033520 & 1.3770 \\
\hline \multicolumn{3}{|c|}{$T=288.15 \mathrm{~K}$} & \multicolumn{3}{|c|}{$T=303.15 \mathrm{~K}$} & \multicolumn{3}{|c|}{$T=318.15 \mathrm{~K}$} \\
\hline 0 & 1.038685 & 2.4198 & 0 & 1.028006 & 1.6008 & 0 & 1.016632 & 1.1485 \\
\hline 0.0512 & 1.040990 & 2.4524 & 0.0507 & 1.030324 & 1.6167 & 0.0501 & 1.018814 & 1.1699 \\
\hline 0.1025 & 1.043039 & 2.4890 & 0.1014 & 1.032407 & 1.6368 & 0.1003 & 1.020996 & 1.1762 \\
\hline 0.1541 & 1.045187 & 2.5230 & 0.1525 & 1.034555 & 1.6596 & 0.1508 & 1.023144 & 1.1874 \\
\hline 0.2058 & 1.047346 & 2.5586 & 0.2037 & 1.036752 & 1.6812 & 0.2015 & 1.025405 & 1.2071 \\
\hline 0.2578 & 1.049447 & 2.6003 & 0.2552 & 1.038870 & 1.7035 & 0.2524 & 1.027545 & 1.2178 \\
\hline 0.3100 & 1.051558 & 2.6285 & 0.3069 & 1.041011 & 1.7395 & 0.3036 & 1.029714 & 1.2364 \\
\hline
\end{tabular}

Table 4. Experimental densities, $\rho$, and viscosities, $\eta$, as function of concentration, $c$, of $\mathrm{NaCl}$ solution in water-1,4-dioxane mixtures with mole fraction of 1,4- dioxane, $x_{\mathrm{D}}=0.20$ at different temperatures.

\begin{tabular}{|c|c|c|c|c|c|c|c|c|}
\hline$c / \mathrm{mol} \mathrm{dm}{ }^{-3}$ & $\rho / \mathrm{g} \mathrm{cm}^{-3}$ & $\eta / \mathrm{mPa} s$ & $c / \mathrm{mol} \mathrm{dm}$ & $\rho / \mathrm{g} \mathrm{cm}^{-3}$ & $\eta / \mathrm{mPa} s$ & $c / \mathrm{mol} \mathrm{dm}^{-3}$ & $\rho / \mathrm{g} \mathrm{cm}^{-3}$ & $\eta / \mathrm{mPa} s$ \\
\hline \multicolumn{3}{|c|}{$T=278.15 \mathrm{~K}$} & \multicolumn{3}{|c|}{$T=293.15 \mathrm{~K}$} & \multicolumn{3}{|c|}{$T=308.15 \mathrm{~K}$} \\
\hline 0 & 1.05074 & 3.5444 & 0 & 1.03920 & 2.2420 & 0 & 1.02715 & 1.5131 \\
\hline 0.0518 & 1.05306 & 3.6031 & 0.0512 & 1.04150 & 2.2680 & 0.0506 & 1.02946 & 1.5392 \\
\hline 0.1036 & 1.05508 & 3.6543 & 0.1025 & 1.04357 & 2.3080 & 0.1013 & 1.03157 & 1.5587 \\
\hline 0.1559 & 1.05718 & 3.7045 & 0.1542 & 1.04572 & 2.3514 & 0.1524 & 1.03371 & 1.5820 \\
\hline 0.2082 & 1.05938 & 3.7568 & 0.2059 & 1.04789 & 2.3745 & 0.2036 & 1.03593 & 1.5963 \\
\hline 0.2608 & 1.06146 & 3.8076 & 0.2580 & 1.04999 & 2.3927 & 0.2551 & 1.03807 & 1.6147 \\
\hline 0.3136 & 1.06355 & 3.8630 & 0.3102 & 1.05211 & 2.4197 & 0.3067 & 1.04022 & 1.6372 \\
\hline \multicolumn{3}{|c|}{$T=283.15 \mathrm{~K}$} & \multicolumn{3}{|c|}{$T=298.15 \mathrm{~K}$} & \multicolumn{3}{|c|}{$T=313.15 \mathrm{~K}$} \\
\hline 0 & 1.04695 & 3.0064 & 0 & 1.03523 & 1.9552 & 0 & 1.02300 & 1.3537 \\
\hline 0.0516 & 1.04926 & 3.0570 & 0.0510 & 1.03754 & 1.9783 & 0.0504 & 1.02530 & 1.3799 \\
\hline 0.1033 & 1.05130 & 3.0984 & 0.1021 & 1.03962 & 2.0019 & 0.1009 & 1.02743 & 1.3936 \\
\hline 0.1553 & 1.05345 & 3.1401 & 0.1536 & 1.04177 & 2.0258 & 0.1518 & 1.02958 & 1.4099 \\
\hline 0.2074 & 1.05560 & 3.1823 & 0.2052 & 1.04395 & 2.0552 & 0.2028 & 1.03181 & 1.4269 \\
\hline 0.2599 & 1.05769 & 3.2230 & 0.2570 & 1.04606 & 2.0740 & 0.2541 & 1.03395 & 1.4398 \\
\hline 0.3125 & 1.05979 & 3.2673 & 0.3090 & 1.04820 & 2.1205 & 0.3055 & 1.03612 & 1.4551 \\
\hline \multicolumn{3}{|c|}{$T=288.15 \mathrm{~K}$} & \multicolumn{3}{|c|}{$T=303.15 \mathrm{~K}$} & \multicolumn{3}{|c|}{$T=318.15 \mathrm{~K}$} \\
\hline 0 & 1.04311 & 2.5757 & 0 & 1.03122 & 1.7027 & 0 & 1.01876 & 1.2145 \\
\hline 0.0514 & 1.04541 & 2.6199 & 0.0508 & 1.03354 & 1.7283 & 0.0502 & 1.02104 & 1.2363 \\
\hline 0.1029 & 1.04746 & 2.6538 & 0.1017 & 1.03562 & 1.7502 & 0.1005 & 1.02323 & 1.2475 \\
\hline 0.1547 & 1.04961 & 2.6891 & 0.1530 & 1.03777 & 1.7746 & 0.1512 & 1.02537 & 1.2618 \\
\hline 0.2067 & 1.05177 & 2.7284 & 0.2044 & 1.03997 & 1.8072 & 0.2020 & 1.02763 & 1.2811 \\
\hline 0.2590 & 1.05387 & 2.7624 & 0.2561 & 1.04208 & 1.8248 & 0.2530 & 1.02977 & 1.2913 \\
\hline 0.3113 & 1.05598 & 2.8034 & 0.3079 & 1.04423 & 1.8561 & 0.3043 & 1.03194 & 1.3102 \\
\hline
\end{tabular}


In present study, the rearranged Jones-Dole equation (3) was applied. By help of the values of relative permittivity ${ }^{16}$ and viscosity (from present study) of the solvent, and limiting molar conductivities ${ }^{16}$ of the $\mathrm{NaCl}\left(\Lambda^{\infty}\right), \mathrm{Na}^{+}$ $\left(\lambda_{+}^{\infty}\right)$, and $\mathrm{Cl}^{-}\left(\lambda_{-}^{\infty}\right)$ at temperature $T$, values of $A$-coefficients were calculated using equation (4). Applied parameters are given in Table 5 and Table S1 in Supporting information, whereas in Table 6 the values of estimated $A$ coefficients are listed.

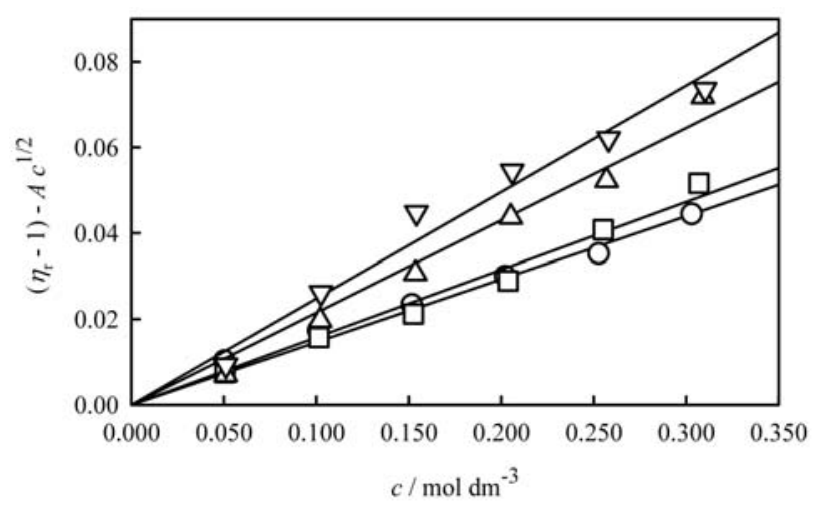

Figure 1. Plots of $\left(\eta_{\mathrm{r}}-1\right)-A c^{1 / 2}$ against $c$ for $\mathrm{NaCl}$ in water-1,4dioxane mixtures at $293.15 \mathrm{~K}$. Experimental values: $\mathrm{O}, x_{\mathrm{D}}=0.05$; $\square, x_{\mathrm{D}}=0.10 ; \triangle, x_{\mathrm{D}}=0.15 ; \nabla, x_{\mathrm{D}}=0.20$. Full lines are linear fits starting from the origin, and from their slopes $B$-coefficients were determined.

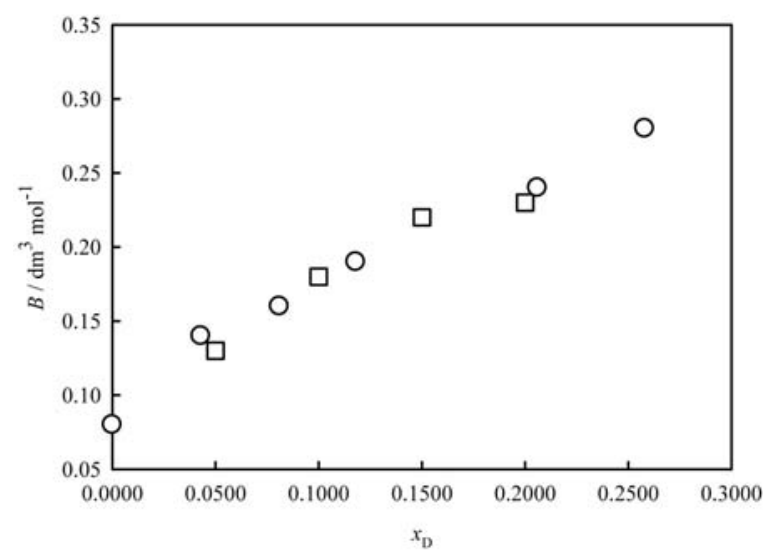

Figure 2. Viscosity $B$-coefficients for $\mathrm{NaCl}$ in water-1,4-dioxane mixtures at 298.15 K: ( $\square$ ), this work; (O), Ref. 20.
In Figure 1 the plot of $\left(\eta_{\mathrm{r}}-1\right)-A c^{1 / 2}$ (rearranged Jones-Dole eq. (3)) in dependence on concentration, $c$, at $293.15 \mathrm{~K}$ is presented. From the slopes viscosity $B$-coefficients were estimated by the assumption that the straight line must cross the origin. Obtained $B$-coefficients are gathered in Table 7 for all investigated systems at all temperatures together with the literature data for $\mathrm{NaCl}$ in water. ${ }^{2}$

A comparison between obtained $B$-coefficients for $\mathrm{NaCl}$ in water-1,4-dioxane mixtures at $298.15 \mathrm{~K}$ with the literature data ${ }^{20}$ shows a reasonable agreement (Figure 2).

Table 6. $A$-coefficients as calculated from Falkenhagen and Vernon eq. (4) for $\mathrm{NaCl}$ in water-1,4-dioxane mixtures at different temperatures. ${ }^{\mathrm{a}}$

\begin{tabular}{lcccc}
\hline $\boldsymbol{T} / \mathbf{K}$ & $\boldsymbol{x}$ D & & & \\
& $\mathbf{0 . 0 5}$ & $\mathbf{0 . 1 0}$ & $\mathbf{0 . 1 5}$ & $\mathbf{0 . 2 0}$ \\
\hline 278.15 & 0.0061 & 0.0071 & 0.0081 & 0.0098 \\
283.15 & 0.0062 & 0.0072 & 0.0083 & 0.0100 \\
288.15 & 0.0063 & 0.0074 & 0.0084 & 0.0101 \\
293.15 & 0.0064 & 0.0075 & 0.0085 & 0.0102 \\
298.15 & 0.0065 & 0.0076 & 0.0086 & 0.0104 \\
303.15 & 0.0067 & 0.0078 & 0.0088 & 0.0106 \\
308.15 & 0.0067 & 0.0079 & 0.0090 & 0.0108 \\
313.15 & 0.0068 & 0.0080 & 0.0091 & 0.0110 \\
318.15 & 0.0069 & 0.0082 & 0.0092 & 0.0112 \\
\hline
\end{tabular}

${ }^{(a)}$ Unit: $A, \mathrm{dm}^{3 / 2} \mathrm{~mol}^{-1 / 2}$

Viscosity $A$-coefficients, listed in Table 6 , are positive at all temperatures. As stated, they depend on the relative permittivity and viscosity of the solvent, but also on the charge and the mobilities of ions and finally on temperature as well. Very small positive $A$-values suggest the presence of relative weak ion-ion interaction (Coulombic forces). From Table 6 thus it can be concluded that Coulombic forces are stronger in systems with higher content of 1,4-dioxane - as it is expected due to lower permittivity of solvent mixture - and at higher temperatures.

The $B$-coefficients are positive (Table 7) at all temperatures and bigger than $A$-coefficients (Table 6), showing the dominance of ions-solvent interaction over ionsions interaction. This finding can be confirmed by small association constant, $K_{\mathrm{A}}$, of $\mathrm{NaCl}$ in water-1,4-dioxane

Table 5. The parameters of Falkenhagen and Vernon eq. (4) for $\mathrm{NaCl}$ in water-1,4-dioxane mixtures at $293.15 \mathrm{~K}$.

\begin{tabular}{|c|c|c|c|c|}
\hline Input data in Eq. $(4)^{a}$ & $x_{\mathrm{D}}=\mathbf{0 . 0 5}$ & $x_{\mathrm{D}}=\mathbf{0 . 1 0}$ & $x_{\mathrm{D}}=0.15$ & $x_{\mathrm{D}}=0.20$ \\
\hline$\lambda_{+}^{\infty} / \mathrm{S} \mathrm{cm}^{2} \mathrm{~mol}^{-1}$ & 33.07 & 25.50 & 21.99 & 19.35 \\
\hline$\lambda_{-}^{\infty} / \mathrm{S} \mathrm{cm}^{2} \mathrm{~mol}^{-1}$ & 48.19 & 36.42 & 31.05 & 26.97 \\
\hline$\Lambda^{\infty} / \mathrm{S} \mathrm{cm}^{2} \mathrm{~mol}^{-1}$ & 81.26 & 61.92 & 53.04 & 46.32 \\
\hline$\varepsilon_{\mathrm{r}}$ & 63.344 & 50.271 & 40.940 & 32.421 \\
\hline
\end{tabular}

${ }^{(a)}$ Values are interpolated or extrapolated from literature data. ${ }^{16}$ 
Table 7. The viscosity $B$-coefficients for $\mathrm{NaCl}$ in water-1,4-dioxane mixtures at different temperatures. ${ }^{\mathrm{a}}$

\begin{tabular}{lccccc}
\hline $\boldsymbol{T} / \mathbf{K}$ & $\mathbf{0 . 0 0}^{\mathbf{b}}$ & $\mathbf{0 . 0 5}$ & $\mathbf{0 . 1 0}$ & $\mathbf{0 . 1 5}$ & $\mathbf{0 . 2 0}$ \\
\hline 278.15 & 0.034 & 0.124 & 0.151 & 0.206 & 0.267 \\
283.15 & 0.053 & 0.129 & 0.152 & 0.235 & 0.260 \\
288.15 & 0.063 & 0.137 & 0.155 & 0.264 & 0.263 \\
293.15 & 0.071 & 0.146 & 0.158 & 0.215 & 0.248 \\
298.15 & 0.080 & 0.129 & 0.174 & 0.221 & 0.231 \\
303.15 & 0.083 & 0.160 & 0.181 & 0.241 & 0.266 \\
308.15 & 0.088 & 0.145 & 0.181 & 0.265 & 0.249 \\
313.15 & 0.093 & 0.141 & 0.190 & 0.271 & 0.234 \\
318.15 & 0.098 & 0.142 & 0.196 & 0.225 & 0.237 \\
\hline
\end{tabular}

${ }^{(a)}$ Unit: $B, \mathrm{dm}^{3} \mathrm{~mol}^{-1} \quad$ (b) Data from Ref. 2.

mixtures with low content of 1,4-dioxane. As reported in ${ }^{16}$ $K_{\mathrm{A}}\left(x_{\mathrm{D}}=0.0575\right)=\sim 5, K_{\mathrm{A}}\left(x_{\mathrm{D}}=0.1104\right)=\sim 8$ and $K_{\mathrm{A}}$ $\left(x_{\mathrm{D}}=0.2000\right)=\sim 24$ at $298.15 \mathrm{~K}$.

At given temperature, the $B$-coefficients mostly follow the order: $B\left(x_{\mathrm{D}}=0.00\right)<B\left(x_{\mathrm{D}}=0.05\right)<B\left(x_{\mathrm{D}}=\right.$ $0.10)<B\left(x_{\mathrm{D}}=0.15\right)<B\left(x_{\mathrm{D}}=0.20\right)$. This increase in the $B$-coefficient with increasing 1,4-dioxane content in the mixture may be attributed to the stronger ions-solvent interactions. ${ }^{28}$

According to classification in literature for water medium, ${ }^{2,30}$ chloride ion is water-structure-breaking ion $(B<0)$, while sodium ion is weak water-structure-making ion $(B>0)$ (or borderline case). Furthermore, it is well known that the $\mathrm{NaCl}$ in water has a structure-breaking nature, thus chloride ion exhibits positive temperature coefficient, $\mathrm{d} B / \mathrm{d} T=0.0014 \mathrm{dm}^{3} \mathrm{~mol}^{-1} \mathrm{~K}^{-1}$, while for sodium ion this value is zero at $298.15 \mathrm{~K}^{2}$

From Table 7 it is evident that viscosity $B$-coefficients for $\mathrm{NaCl}$ in solutions with $x_{\mathrm{D}}=0.05,0.10$, and 0.15 show the trend of an increase with the temperature $(\mathrm{d} B / \mathrm{d} T$ $>0$ ) what can be attributed to a structure-breaking nature of $\mathrm{NaCl}$ in these systems. Namely, with raising temperature hydrogen bonds are breaking and thus less structured water can be influenced by the ionic solute. ${ }^{20}$

Small change of the B-value with temperature for $x_{\mathrm{D}}$ $=0.20$ may be ascribed to the weakening of the ions-solvent interactions in comparison to solvent-solvent interactions. It is worth to mention, that water-1,4-dioxane binary mixtures show maximum in viscosity at $x_{\mathrm{D}} \approx$ $0.25^{26,27,31}$ which can be ascribed to the strong interaction between 1,4-dioxane and water molecules.

\section{Conclusions}

In present work, the $B$-coefficients of $\mathrm{NaCl}$ in water-1,4-dioxane binary mixtures in the temperature range from 278.15 to $318.15 \mathrm{~K}$ in four solvent mixtures with mole fraction of 1,4-dioxane, $x_{\mathrm{D}}=0.05,0.10,0.15$ and 0.20 , were determined by help of the rearranged Jones-
Dole equation, $\left(\eta_{\mathrm{r}}-1\right)-A c^{1 / 2}=B c$, and experimental data on viscosity, whereas the viscosity $A$-coefficients have been calculated from Falkenhagen and Vernon theory.

All obtained values of $B$-coefficients are positive and bigger than values of $A$ in all solvent mixtures suggesting that ions-solvent are prevailing upon ion-ion interactions, what is agreement with small association constants for $\mathrm{NaCl}$ in these mixtures as obtained from conductivity measurement and reported in literature. ${ }^{16}$

Whereas for $\mathrm{NaCl}$ in solutions with $x_{\mathrm{D}}=0.05,0.10$, and 0.15 it mainly states that $\mathrm{d} B / \mathrm{d} T>0$, whereas - due to weak dependence of $B$ on the temperature - this can be not assumed for $x_{\mathrm{D}}=0.20$. It can be concluded that in solutions with lower content of 1,4-dioxane the structurebreaking nature of $\mathrm{NaCl}$ is prevailing and ions-solvent interactions are dominant. With increasing content of 1,4dioxane the solvent-solvent interactions become more important, resulting finally also in a maximum in viscosity at $x_{\mathrm{D}} \approx 0.25^{26,27,31}$ which can be attributed to the strong interaction between 1,4-dioxane and water molecules.

It would be interesting to carry out this kind of research on the systems with higher content of 1,4-dioxane in the solvent. But according to the literature ${ }^{16}$ the association of $\mathrm{NaCl}$ increases dramatically with increasing amount of 1,4-dioxane and thus model, assuming negligible ion association applied in this study, can not be used.

\section{Acknowledgments}

T.J. is grateful to Erasmus mobility grant enabling the research cooperation at University of Ljubljana and Dr. Bojan Šarac for all his help. The financial support from the Slovenian Research Agency through Grant No. P1-0201 and J1-0103-4148 is gratefully acknowledged. M. B.-R. would like to acknowledge Professor Dr. Jože Koller as a kind co-worker.

\section{References}

1. G. Hefter, P. M. May, P. Sipos, A. Stanley, J. Mol. Liq. 2003, 103-104, 261-273. http://dx.doi.org/10.1016/S0167-7322(02)00145-9

2. H. D. B. Jenkins, Y. Marcus, Chem. Rev. 1995, 95, 26952724. http://dx.doi.org/10.1021/cr00040a004

3. J. M. McDowall, N. Martinus, C. A. Vincent, J. Chem. Soc., Faraday Trans. I 1976, 72 654-660. http://dx.doi.org/10.1039/f19767200654

4. J. Doménech, S. Rivera, Z. Phys. Chem. 1983, 136, 153-161. http://dx.doi.org/10.1524/zpch.1983.136.136.153

5. N. G. Tsierkezos, I. E. Molinou, Z. Phys. Chem. 2004, 218, 211-217. http://dx.doi.org/10.1524/zpch.218.2.211.25924

6. M. M. Palaiologou, I. E. Molinou, N. G. Tsierkezos, J. Chem. Eng. Data 2002, 47, 1285-1289.

http://dx.doi.org/10.1021/je020063s 
7. P. S. Nikam, V. P. Sonawane, Int. J. Chem. Sci. 2003, 1, 210-214.

8. N. P. Singh, M. M. Singh, P. K. Tikoo, Aust. J. Chem. 1977, 30, 2303-2305. http://dx.doi.org/10.1071/CH9772303

9. P. Haldar, B. Das, J. Mol. Liq. 2008, 138, 88-92. http://dx.doi.org/10.1016/j.molliq.2007.09.002

10. L. Werblan, A. Rotowska, S. Minc, Electrochim. Acta 1971, 16, 41-59. http://dx.doi.org/10.1016/0013-4686(71)85127-7

11. M. Huque, I. A. Siddiquey, Md. N. Uddin, J. Chem. Thermodynamics 2006, 38, 1474-1478. http://dx.doi.org/10.1016/j.jct.2006.01.002

12. S. Thirumaran, K. Sathish, Res. J. Chem. Sci. 2011, 1(8), 6371.

13. N. G. Tsierkezos, I. E. Molinou, Z. Phys. Chem. 2002, 216, 961-970. http://dx.doi.org/10.1524/zpch.2002.216.8.961

14. P. K. Kipkemboi, A. J. Easteal, Indian J. Chem. 2002, 41A, 1139-1145.

15. J. Barthel, H. Krienke, W. Kunz, Physical Chemistry of Electrolyte Solutions - Modern Aspects, Darmstadt, Steinkoppf, and Springer, NewYork, 1998.

16. M. Bešter-Rogač, R. Neueder, J. Barthel, J. Solution Chem. 1999, 28, 1071-1086. http://dx.doi.org/10.1023/A:1022625310402

17. M. Bešter-Rogač, R. Neueder, J. Barthel, J. Solution Chem. 2000, 29, 51-61. http://dx.doi.org/10.1023/A:1005114500861

18. M. Bešter-Rogač, N. Hauptman, J. Barthel, J. Mol. Liq. 2007, 131-132, 29-35.

http://dx.doi.org/10.1016/j.molliq.2006.08.024
19. M. Bončina and M. Bešter-Rogač, Acta Chim. Slov. 2012, 59, 536-540.

20. R. M. Fuoss and A. F. Reynolds, Isr. J. Chem. 1973, 11, 201-210. http://dx.doi.org/10.1002/ijch.197300022

21. N. C. Das, P. B. Das, Electrochim. Acta 1980, 25, 725-728. http://dx.doi.org/10.1016/0013-4686(80)87085-X

22. H. S. Harned, B. B. Owen, The Physical Chemistry of Electrolytic Solutions, $3^{\text {rd }}$ Edition, Reinhold Publishing Corporation, New York, 1963, p. 236.

23. M. R. Cannon, R. E. Manning, J. D. Bell, Anal. Chem. 1960, 32, 355-358. http://dx.doi.org/10.1021/ac60159a015

24. IUPAC, Recommended Reference Materials for Realization of Physicochemical Properties. Section: Viscosity, Pure Appl. Chem. 1980, 52, 2395-2404.

25. O. Kratky, H. Leopold, H. N. Stabinger, Z. Angew. Phys. 1969, 27, 273-277.

26. L.-M. Omota, O. Iulian, O. Ciocirlan, I. Nita, Rev. Roum. Chim. 2008, 53, 977-988.

27. L.-M. Omota, O. Iulian, F. Omota, O. Ciocirlan, Rev. Roum. Chim. 2009, 54, 63-73.

28. B. Gurung, M. N. Roy, Solute-Solvent Interactions in Industrially Important Solvent Media, VDM Verlag Dr. Müller Aktiengesellschaft \& Co. KG, Saarbrücken, 2010, pp. 13-39.

29. M. Kaminsky, Discuss. Faraday Soc. 1957, 24, 171-179. http://dx.doi.org/10.1039/df9572400171

30. Y. Marcus, Pure Appl. Chem. 2010, 82, 1889-1899. http://dx.doi.org/10.1351/PAC-CON-09-07-02

31. G. Ahn-Ercan, H. Krienke, G. Schmeer, J. Mol. Liq. 2006, 129, 75-79. http://dx.doi.org/10.1016/j.molliq.2006.08.004

\section{Povzetek}

Izmerili smo viskoznosti raztopin $\mathrm{NaCl} v$ mešanicah vode in 1,4-dioksana (z molskim deležem 1,4-dioksana, $x_{\mathrm{D}}=0.05$, $0.10,0.15$ in 0.20$)$ v temperaturnem območju med 278.15 in $318.15 \mathrm{~K}$. Dobljene vrednosti relativnih viskoznosti smo analizirali in razložili s pomočjo Jones-Dolove enačbe $\left(\eta_{r}-1\right)-A c^{1 / 2}=B c$. Koeficiente $A$ smo pridobili z uporabo Falkenhagenove in Vernonove teorije, koeficiente $B$ pa smo določili z linearno regresijo. Dobljene vrednosti $B$-koeficientov za $\mathrm{NaCl}$ v proučevanih topilih so pozitivne pri vseh temperaturah. Podatke smo primerjali s podatki iz literature za $\mathrm{NaCl}$ v vodi pri različnih temperaturah ter v mešanicah vode in 1,4-dioksana pri $298.15 \mathrm{~K}$ ter jih interpretirali s pomočjo možnih interakcij v preiskovanih raztopinah. 